\title{
Alongamento estático versus conceito Mulligan - efeitos crônicos no treino de flexibilidade em ginastas
}

\author{
Static stretch versus Mulligan Concept - long-term effects \\ in gymnast's flexibility
}

\author{
Manuela Karloh \\ Raquel Petry dos Santos \\ Maria Helena Kraeski \\ Thiago Sousa Matias \\ Anderson Simas Frutuoso
}

1 Universidade do Estado de Santa Catariana. Mestrado em Ciências do Movimento Humano. Florianópolis, SC, Brasil.

2 Universidade do Estado de Santa Catariana. Florianópolis, SC, Brasil.

Recebido em 17/04/09 Revisado em 21/06/09 Aprovado em 21/12/09
Resumo - A flexibilidade é uma das valências físicas essenciais para a prática da Ginástica Rítmica (GR). Este estudo tem por objetivo comparar as adaptações crônicas do treino de flexibilidade em função de duas técnicas: o alongamento estático e a técnica Mulligan Long Leg Traction, no movimento de extensão do quadril, em atletas de GR. Participaram do estudo oito atletas do sexo feminino, com média de idade de 13,25 $\pm 0,89$ anos, divididas em dois grupos. O Grupo 1 foi submetido à técnica Mulligan e o Grupo 2 ao alongamento estático. $\mathrm{O}$ treinamento de flexibilidade teve duração de seis semanas, foi realizado duas vezes por semana e constitui-se de duas repetições de 30 segundos para cada membro inferior. Utilizou-se a fotogrametria para avaliar a amplitude de movimento (ADM) de extensão do quadril. Realizou-se a avaliação da ADM antes do início e após seis semanas de treinamento. Utilizou-se estatística descritiva e inferencial para o tratamento dos dados. $O$ aumento da ADM foi estatisticamente significativo em ambos os membros inferiores no Grupo 1, e no MID no Grupo 2. Após seis semanas de treinamento, o ganho de ADM do Grupo 1 foi de $6,25^{\circ} \pm 2,75^{\circ}$ no MIE e, $525^{\circ} \pm 2,63^{\circ}$ no MID; e do grupo 2 foi $6,75^{\circ} \pm 4,64^{\circ}$ no MIE e 5,5 $\pm 3,41^{\circ}$ no MID. Ao comparar as técnicas executadas, em relação ao ganho de ADM, não houve diferença estatisticamente significativa. Conclui-se que, após as seis semanas de treinamento, as duas técnicas propostas promoveram aumento estatisticamente significativo na amplitude de movimento.

Palavras-chave: Exercícios de Alongamento Muscular; Amplitude de Movimento Articular; Ginástica.

Abstract - Flexibility is one of the most important physical aspects in Rhythmic Gymnastics $(R G)$. The purpose of the study was to compare long-term changes in flexibility of hip extension in athletes of RG in function of two techniques: the static stretch and Mulligan's Long Leg Traction. Participated eight female athletes with an average age of 13,25 0,89 years old, divided into two groups. Group 1 performed Mulligan technique and Group 2 performed static stretch. Flexibility training lasts for six weeks. It was executed 2 times a week, and was composed by 2 repetitions of 30 seconds for each lower limb. Photogrammetry was used to assess the range of motion (ROM) of hip extension. ROM was assessed before and after six weeks of training. Descriptive and inferential statistics were used to analyze the data. The increase of ROM was statistically significant in both lower limbs in Group 1, and in right lower limb (RLL) in Group 2. After six weeks of training the increase of $R O M$ in Group 1 was $6,25^{\circ} \pm 2,75^{\circ}$ in left lower $\operatorname{limb}(L L L)$ and $5,25^{\circ} \pm 2,63^{\circ}$ in RLL, and the increase in Group 2 was $6,75^{\circ} \pm 4,64^{\circ}$ in $L L L$ and $5,5^{\circ} \pm 3,41^{\circ}$ in RLL. Comparing the two executed techniques, in relation to the increase of $R O M$, there were no statistically significant differences. We conclude that after six weeks of training the two proposed techniques have showed increases in range of motion.

Key words: Muscle Stretching Exercises; Range of Motion, Articular; Gymnastics. 


\section{INTRODUÇÃO}

A Ginástica Rítmica (GR) é uma modalidade esportiva que se destaca pela elegância e beleza dos movimentos e requer um alto nível de desenvolvimento de algumas qualidades físicas, objetivando a perfeição técnica na execução dos elementos corporais e manejo dos aparelhos'.

Dentre as diferentes qualidades físicas inerentes à ginástica, a flexibilidade exerce um papel decisivo nesta modalidade, além de ser um elemento corporal previsto pelo Código de Pontuação da Federação Internacional de Ginástica ${ }^{2}$. Os gestos esportivos deste esporte caracterizam-se pela ampla utilização das articulações, principalmente, dos movimentos de extensão da coluna vertebral e do quadril ${ }^{3}$.

O aprimoramento da flexibilidade desenvolvese por diferentes técnicas, dentre as quais estão: o alongamento dinâmico ou balístico, alongamento estático ou passivo e a Facilitação Neuromuscular Proprioceptiva ${ }^{4,5}$.

O alongamento estático é o método mais comum para o ganho de flexibilidade e oferece vantagens quando comparado às outras duas formas de alongamento devido à facilidade de execução e ao baixo potencial de dano tecidual ${ }^{4-6}$.

Além dos métodos descritos acima, encontra-se na literatura a descrição da utilização de técnicas do conceito Mulligan para o aprimoramento da flexibilidade ${ }^{7,8}$. Estudos demonstram a aplicação de técnicas denominadas Long Leg Traction, que consistem na associação de alongamentos estáticos com trações, visando aumentar a amplitude de movimento (ADM) dos membros inferiores ${ }^{7,8}$.

Assim, esse estudo tem como objetivo investigar e comparar as adaptações crônicas do treino de flexibilidade da articulação do quadril no movimento de extensão, em atletas de Ginástica Rítmica, em função de duas técnicas de alongamento: o alongamento estático e a técnica Mulligan - Long Leg Traction.

\section{PROCEDIMENTOS METODOLÓGICOS}

Esta é uma série de casos, de natureza quaseexperimental e caracteriza-se por uma abordagem quantitativa9. O estudo foi aprovado pelo Comitê de Ética em Pesquisa em Seres Humanos - CEP/ UDESC. Os responsáveis pelas atletas receberam as informações referentes à execução do estudo e assinaram o Termo de Consentimento Livre e Esclarecido.

\section{Participantes}

Participam do estudo todas as atletas com idade entre 12 e 16 anos que compõem a equipe de GR ADIEE/UDESC (Florianópolis/SC), totalizando 8 ginastas.

A escolha dos sujeitos foi realizada intencionalmente e por conveniência. Os critérios de inclusão foram: praticantes de GR, com idade entre 12 e 16 anos. Determinou-se como critério de exclusão a presença de lesões músculo-esqueléticas, no período do estudo, que influenciassem ou limitassem a ADM do quadril. Entretanto nenhuma atleta foi acometida por lesões no período do estudo.

Instrumentos da Avaliação e Coleta dos Dados Para avaliar a ADM de extensão do quadril, utilizou-se a fotogrametria ${ }^{10}$. Os ângulos articulares foram quantificados pelo software AutoCAD 2007. Para o registro fotográfico, utilizou-se uma máquina fotográfica digital, Sony®DSCT5, 5.1 megapixels de resolução, lente Carl Zeiss Vario-Tessar fixada sobre um tripé Vivitar ${ }^{\circledR}$ de 1,30 metros de altura, posicionado a 3 metros de distância do local, no qual as atletas foram posicionadas, de modo que a divergência produzida pela lente convencional da câmera não interferisse nas mensurações dos ângulos articulares de modo significativo ${ }^{11}$. Foi utilizada uma cinta Mulligan ${ }^{\circledR}$ fixada na parte proximal da coxa das atletas para auxiliar a aplicação de uma das técnicas propostas.

\section{Avaliação Inicial}

A avaliação inicial foi realizada antes de iniciar os treinos de flexibilidade, e foi constituída por: anamnese (iniciais do nome da atleta, idade, tempo de prática de GR, membro inferior dominante) e avaliação da ADM de extensão do quadril por meio da fotogrametria. Para tal, as atletas foram posicionadas sobre um cavalo de saltos da Ginástica Artística, em seguida, realizou-se a marcação com etiquetas adesivas colocadas nos pontos de referência articular: epicôndilo lateral do fêmur, trocânter maior do fêmur e lóbulo da orelha ${ }^{12}$.

$\mathrm{O}$ ângulo mensurado correspondeu ao movimento de extensão do quadril e constitui-se da intersecção da reta formada pelos pontos epicôndilo lateral do fêmur e trocânter maior do fêmur (reta $\mathrm{A}$ Figura 1), com a reta que parte do lóbulo da orelha, traçada paralelamente à superfície do local onde a atleta foi posicionada (reta B Figura 1). Para a mensuração, utilizou-se a posição do Teste de Thomas modificado ${ }^{13}$. O movimento de extensão do quadril foi executado passivamente pelo avaliador até o 
ponto em que a atleta conseguisse manter a coluna lombar retificada, em contato com a superfície do cavalo de saltos. A articulação do joelho permaneceu livre. O registro fotográfico deu-se na posição de máxima ADM passiva da extensão do quadril13 .

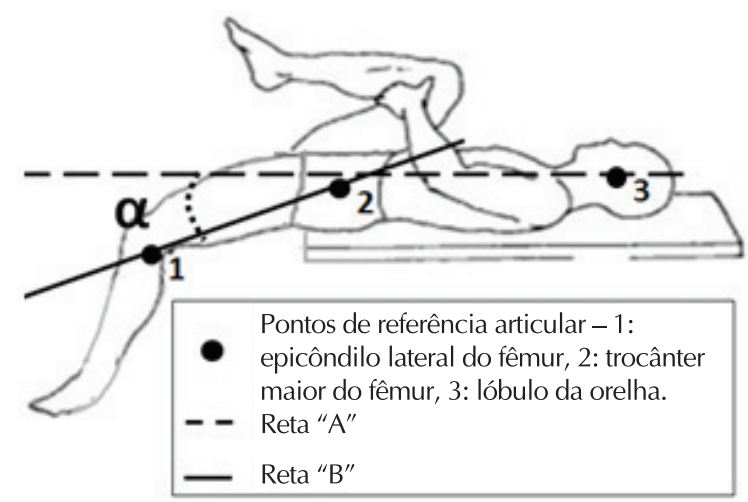

Figura 1. Mensuração do ângulo de extensão do quadril (adaptado de McCreary et al. ${ }^{12}$ )

Treinos de Flexibilidade - Intervenção nos Grupos 1 e 2

Para a aplicação dos protocolos propostos, as atletas foram divididas por ranqueamento de acordo com a ADM de extensão do quadril encontrada na avaliação inicial. Sendo assim, as atletas receberam uma classificação de "um” a "oito"; o valor "um" correspondeu ao maior resultado no teste de flexibilidade e o valor "oito" ao menor. As atletas foram pareadas de acordo com a sua classificação, por exemplo, atletas 1 e 2, 3 e 4 e assim sucessivamente. Em seguida, em cada dupla, foi realizado um sorteio para designar qual atleta da dupla pertence ao grupo 1 ou 29 .

A designação do método de treinamento dos grupos 1 e 2 foi realizada por sorteio. $\bigcirc$ grupo 1 foi composto por quatro atletas submetidas ao treino de flexibilidade, utilizando apenas a técnica Mulligan - Long Leg Traction (alongamento estático associado à tração do segmento) para extensão do quadril (Figura 2-A). O grupo 2 foi composto por quatro atletas que foram submetidas ao treino de flexibilidade, utilizando somente o alongamento estático (Figura 2-B).

As coletas de dados foram realizadas no período de 15/07 a 15/09/2008, no período da tarde. Não houve controle das variáveis temperatura, umidade e pressão uma vez que as coletas de dados ocorreram no ginásio de treinamentos da equipe. Realizaramse 11 treinos de flexibilidade para cada grupo. Esses aconteceram duas vezes na semana, sempre após o aquecimento das atletas, no qual se executou uma rotina de exercícios específicos da modalidade, com cerca de 50 minutos de duração ${ }^{1}$. Em cada dia de treinamento, realizaram-se duas repetições da intervenção proposta (Mulligan ou alongamento estático) em cada membro inferior, com duração de 30 segundos e repouso de 30 segundos entre as séries ${ }^{5,6,14,15}$. Os treinos foram aplicados por um pesquisador, com experiência na modalidade e que domina as duas técnicas em questão.

A

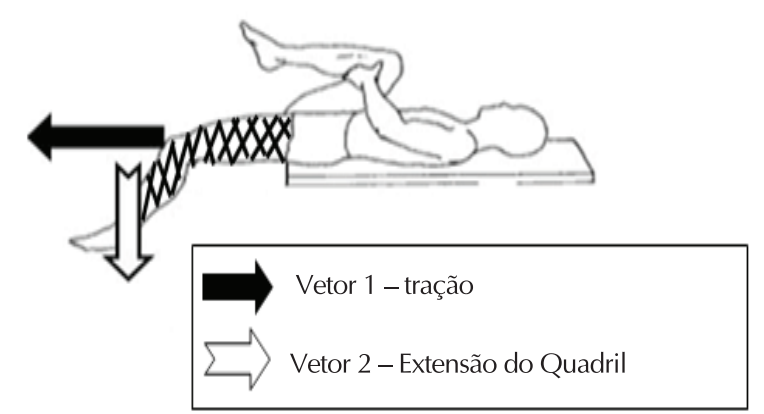

Figura 2. (A) Mulligan Long Leg Traction (McCreary et al. ${ }^{12}$ ); Alongamento Estático'.

\section{Avaliação Final}

Após a aplicação das seis semanas de treinamento de flexibilidade (11 treinos), realizou-se a avaliação final da ADM de extensão do quadril. Esta foi executada pelo mesmo avaliador e de maneira idêntica à avaliação inicial.

\section{Análise dos resultados}

Os dados foram sistematizados no programa SPSS versão 16.0. Para a análise dos resultados, utilizou-se estatística descritiva - mínimo, máximo, média e desvio padrão. Antes dos testes inferenciais, foi realizada uma análise exploratória dos dados, verificaram-se os valores de assimetria e curtose, bem como, a normalidade com o teste de Shapiro-Wilk. Como a distribuição dos dados foi normal, para comparar os grupos, foi utilizado o teste " $\mathrm{t}$ " de student para amostras independentes e para o delineamento pré e pós teste intra grupo foi utilizado o teste " $t$ " para amostras pareadas. O nível de significância foi de p<0,05.

\section{RESULTADOS}

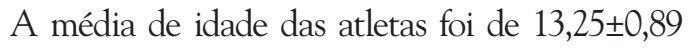
anos. $\mathrm{O}$ tempo de prática médio no esporte foi de $5,44 \pm 2,26$ anos. Com relação à dominância, em sete das oito atletas o membro inferior direito é o dominante.

Os indivíduos do grupo 1 foram submetidos ao treino de flexibilidade, utilizando apenas a técnica Mulligan-Long Leg Traction. Os valores referentes às amplitudes de movimento antes e após o período de treinamento estão apresentados na tabela 1 . O aumento na 
ADM de extensão do quadril nos indivíduos do grupo 1 foi estatisticamente significativo para ambos os membros inferiores. Após as seis semanas de treinamento, as atletas do grupo 1 apresentaram um ganho de ADM de $6,25^{\circ} \pm 2,75^{\circ}$ no membro inferior esquerdo (MIE) e $5,25^{\circ} \pm 2,63^{\circ}$ no membro inferior direito (MID).

Os indivíduos do Grupo 2 foram submetidos ao treino de flexibilidade, utilizando somente o alongamento estático. $\mathrm{O}$ aumento na ADM das atletas do Grupo 2 foi estatisticamente significativo somente no MID. $\mathrm{O}$ ganho de ADM do grupo 2 foi $6,75^{\circ} \pm 4,64^{\circ}$ no MIE e $5,5^{\circ} \pm 3,41^{\circ}$ no MID. As ADM inicial e final estão descritas na tabela 2.

Ao comparar os ganhos da ADM após seis semanas de treinamento de flexibilidade de acordo com a técnica executada - Long Leg Traction (Grupo 1) ou alongamento estático (Grupo 2), observou-se que o alongamento estático apresentou valores discretamente superiores. A diferença das técnicas no MIE foi de $0,50^{\circ}$ e no MID de $0,25^{\circ}$, neste caso, esta diferença não foi estatisticamente significativa (tabela 3).

\section{DISCUSSÃO}

As atletas apresentaram ADM inicial do movimento de extensão da articulação coxofemoral de $31,38^{\circ} \pm 5,26^{\circ}$ no MIE e $30,35^{\circ} \pm 4,04^{\circ}$ no MID Steinberg et al..$^{16}$ analisaram a ADM de extensão do quadril em bailarinos e não bailarinos de diversas faixas etárias. Nos bailarinos de 12 a 16 anos, a amplitude de movimento encontrada variou de $30^{\circ}$ a $35^{\circ}$, valores similares aos encontrados em nosso estudo. Enquanto para os não-bailarinos, os valores foram próximos a $20^{\circ}$. Kirby et al. ${ }^{17}$ avaliaram 60 ginastas profissionais e 35 indivíduos não atletas. Concluíram que as ginastas apresentavam valores de ADM de extensão de quadril estatisticamente superiores às não atletas. $\mathrm{O}$ estudo de L'Hermette et al. ${ }^{18}$ analisou a flexibilidade do quadril de 97 atletas de handebol, sendo que estas apresentaram em média $6^{\circ}$ de extensão de quadril.

Assim, observou-se que a flexibilidade é uma característica marcante de esportes que exigem componentes estéticos e elásticos, dentre os quais está a GR. Já para outros esportes e para indivíduos não atletas a flexibilidade não é uma característica marcante uma vez que não é uma qualidade física crucial para o rendimento.

Adaptações Crônicas ao Alongamento - Grupo 1 Os resultados demonstraram o aumento significativo na ADM de extensão do quadril nas atletas do Grupo 1, submetidas ao treinamento de flexi-

Tabela 1. Amplitude de Movimento Inicial e Final do MIE e MID do grupo 1(Mulligan Long Leg Traction)

\begin{tabular}{lccccc}
\hline & Mínimo & Máximo & Média & Desvio Padrão & $p$ \\
\hline Avaliação inicial do MIE & $25^{\circ}$ & $38^{\circ}$ & $31,75^{\circ}$ & $5,85^{\circ}$ & 0,020 \\
Avaliação final do MIE & $28^{\circ}$ & $44^{\circ}$ & $38^{\circ}$ & $7,35^{\circ}$ & \\
Avaliação inicial do MID & $26^{\circ}$ & $35^{\circ}$ & $31,5^{\circ}$ & $4,04^{\circ}$ & 0,028 \\
Avaliação final do MID & $29^{\circ}$ & $40^{\circ}$ & $36,75^{\circ}$ & $5,19^{\circ}$ & 0 \\
\hline
\end{tabular}

MIE: membro inferior esquerdo/ MID: Membro inferior direito

Tabela 2. Amplitude de Movimento Inicial e Final do MIE e MID do grupo 2 (Alongamento Estático)

\begin{tabular}{lccccc}
\hline & Mínimo & Máximo & Média & Desvio Padrão & $p$ \\
\hline Avaliação inicial do MIE & $27^{\circ}$ & $39^{\circ}$ & $31^{\circ}$ & $5,48^{\circ}$ & 0,062 \\
Avaliação final do MIE & $29^{\circ}$ & $44^{\circ}$ & $37,75^{\circ}$ & $6,5^{\circ}$ & \\
Avaliação inicial do MID & $25^{\circ}$ & $34^{\circ}$ & $29^{\circ}$ & $4,24^{\circ}$ & 0,049 \\
\hline Avaliação final do MID & $27^{\circ}$ & $38^{\circ}$ & $34,5^{\circ}$ & $5,06^{\circ}$ & 0,04 \\
\hline
\end{tabular}

MIE: membro inferior esquerdo/ MID: Membro inferior direito.

Tabela 3. Ganho de amplitude de movimento após o treinamento (adaptações crônicas ao alongamento).

\begin{tabular}{lcccc}
\hline & Designação dos Grupos & Média & Desvio Padrão & $p$ \\
\hline $\begin{array}{l}\text { Ganho de ADM do membro } \\
\text { inferior esquerdo }\end{array}$ & Mulligan & $6,25^{\circ}$ & $2,7^{\circ}$ & 0,861 \\
\cline { 2 - 3 } $\begin{array}{l}\text { Ganho de ADM do membro } \\
\text { inferior direito }\end{array}$ & Alongamento Estático & $6,75^{\circ}$ & $4,64^{\circ}$ & $2,63^{\circ}$ \\
\hline
\end{tabular}

ADM: Amplitude de Movimento. 
bilidade, utilizando somente a técnica Mulligan Long Leg Traction.

Apesar dos efeitos crônicos das técnicas de tração de Mulligan no ganho de flexibilidade não terem sido descritos na literatura, alguns estudos têm demonstrado a eficiência dessas técnicas no ganho imediato de amplitude de movimento, principalmente, na articulação coxofemoral. Hall et al. ${ }^{8}$ demonstraram um aumento de $13,3^{\circ}$ na flexão de quadril após a aplicação de três repetições da mesma técnica utilizada no presente estudo ${ }^{8}$. Mais recentemente, os mesmos autores descrevem um aumento de $11^{\circ}$ na flexão do quadril imediatamente após a intervenção através da mesma técnica9 .

O estudo de Mosler et al. ${ }^{20}$ utiliza uma técnica de tração com auxílio de uma cinta, semelhante à utilizada no presente estudo. Os autores demonstraram, após oito intervenções (quatro semanas), o aumento estatisticamente significativo na amplitude de movimento do quadril, em atletas de pólo aquático, submetidos à tração do quadril com auxílio de uma cinta, aliada a outras terapias manuais (desativação de pontos-gatilhos, massagem por fricção, alongamentos). O protocolo proposto foi aplicado duas vezes na semana, por quatro semanas, com duração de 45 minutos por sessão.

Adaptações Crônicas ao Alongamento- Grupo 2 As atletas do presente estudo, que realizaram o treino de flexibilidade, utilizando somente o alongamento estático, demonstraram incremento na amplitude de movimentos após as seis semanas de treinamento. Porém, o aumento foi considerado estatisticamente significativo somente no membro inferior direito. Este dado pode estar relacionado ao fato de a amplitude de movimento inicial do membro inferior direito ser inferior à do membro inferior esquerdo.

Os efeitos, em longo prazo, do alongamento estático na amplitude de movimento têm sido estudados por diversos autores ${ }^{21-23}$. Gajdosik reporta que os indivíduos do grupo teste (12 indivíduos não atletas e saudáveis) submetidos a sessões diárias de alongamento, compostas por 10 repetições de 15 segundos, com 15 segundos de repouso entre as repetições, por um período de três semanas, demonstraram incremento de $13,4^{\circ}$ na amplitude de movimento de flexão do quadril ${ }^{24}$. Bandy e Irion ${ }^{5}$ e Bandy et al..$^{6}$ relatam o aumento de $12,5^{\circ}$ e $10,05^{\circ}$, respectivamente, na amplitude de movimento de flexão do quadril após um programa de flexibilidade através de alongamentos estáticos com duração de seis semanas (três repetições de 30 segundos). Outro estudo também observou o incremento na flexibilidade dos membros inferiores após um período de 13 semanas. Os autores encontraram um aumento de $7^{\circ}$ na amplitude de movimento de flexão do quadril, no grupo submetido a um programa de alongamento estático realizado três vezes por dia, sendo que cada sessão constituiu-se de cinco repetições de 30 segundos $^{15}$.

\section{Adaptações Crônicas - Grupo 1 versus Grupo 2}

O aumento da amplitude de movimento, após completar o programa de treinamento, no presente estudo, corrobora com os achados de Roberts e Wilson ${ }^{4}$ e Bandy e Irion ${ }^{5}$. Os estudos observaram o aumento significativo nas amplitudes de movimento ativa e passiva dos membros inferiores de adultos jovens após cinco semanas de treinamento de flexibilidade, através de alongamentos estáticos, aplicados três vezes por semana, com três repetições de 15 segundos e repouso de 15 segundos entre as séries ${ }^{4}$ e após seis semanas de treinamento, através de alongamentos estáticos realizados cinco vezes por semana, com duração de 30 segundos apenas uma vez por dia 5 .

Existem controvérsias na literatura a respeito dos mecanismos pelos quais os exercícios de alongamento promovem alterações crônicas na amplitude de movimento. Três mecanismos têm sido descritos como responsáveis pelo aumento permanente na amplitude de movimento, após um programa de exercícios de alongamento: as alterações nas propriedades viscoelásticas do tecido muscular ${ }^{2324}$; o efeito neurofisiológico através do aumento na tolerância ao alongamento ${ }^{21,26}$; e a remodelação tecidual ${ }^{28-30}$.

Winters et al..$^{23} \mathrm{e}$ Gajdosik ${ }^{24}$ sugerem que o efeito crônico no ganho da amplitude de movimento, em resposta ao alongamento, decorre de alterações nas propriedades viscoelásticas dos tecidos envolvidos. Esse mecanismo determina que quando uma unidade músculo-tendínea é alongada e mantida a um comprimento fixo por um determinado período, resulta em uma diminuição da tensão ao longo do tempo, levando a alterações no comprimento muscular ${ }^{14}$.

Todavia, segundo De Deyne, as alterações nas propriedades viscoelásticas do músculo explicam somente as adaptações agudas ao alongamento ${ }^{25}$. Porém, esse mecanismo falha ao explicar as alterações crônicas ao alongamento ${ }^{26}$. Magnusson relata que as propriedades viscoelásticas do tecido muscular são afetadas por exercícios de alongamento, entretanto, retornam aos valores iniciais dentro de 1 hora $^{21}$. Magnusson explica que se as adaptações viscoelásticas do tecido fossem o mecanismo pelo qual há o aumento na amplitude de movimento após um programa de flexibilidade, a resposta 
esperada seria a redução na rigidez muscular e na energia. Porém, os dados encontrados por ele demonstraram que esses dois parâmetros permaneceram inalterados após o programa de alongamento, ou seja, não ocorrem mudanças permanentes nas propriedades viscoelásticas do tecido muscular após um programa de alongamento composto por duas sessões diárias e com duração de 20 dias $^{21}$.

Magnusson et al. atribuem o incremento na amplitude de movimento ao aumento na tolerância ao alongamento ${ }^{26}$. O mecanismo que explica a alteração na tolerância não é conhecido ${ }^{21}$, entretanto, sugere-se que os mecanismos periféricos, através dos receptores dos músculos, tendões e articulações possam estar envolvidos. $\mathrm{O}$ estudo de Guissard e Duchateau $^{27}$ demonstrou o aumento da ADM de dorsiflexão do tornozelo após um programa de seis semanas de alongamento estático do tríceps sural, realizado diariamente por 10 minutos. Os autores sugerem que a redução no input neural aos motoneurônios possa contribuir parcialmente para o ganho de flexibilidade. Encontraram a diminuição da atividade tônica reflexa dos reflexos $\mathrm{T}$ e $\mathrm{H}$ após o programa. Esses resultados sugerem a diminuição da excitabilidade e/ou da transmissão sináptica das fibras Ia aferentes ao motoneurônio; ou também, a redução na sensibilidade dos fusos musculares.

Outros autores defendem que para ocorrer alterações duradouras no comprimento muscular são necessárias modificações na estrutura do músculo, ou seja, remodelação tecidual ${ }^{28-30}$. Estudos em animais demonstram que aumentos crônicos no comprimento do músculo provavelmente resultem do remodelamento do tecido muscular, ocorrendo alterações no comprimento ${ }^{29}$, peso das fibras musculares ${ }^{30}$, número de sarcômeros em série ${ }^{29,30} \mathrm{e}$ aumento da área de secção transversa ${ }^{29,30}$.

O estudo de Coutinho et al. ${ }^{29}$ avaliou o efeito de um protocolo de alongamento aplicado no músculo sóleo de ratos submetidos à imobilização do mesmo. Os autores demonstraram o aumento significativo no comprimento $(5 \pm 2 \%)$, no número de sarcômeros em série $(4 \pm 4 \%)$ e na área de secção transversa do músculo no grupo de ratos submetidos a um programa de três semanas de alongamento estático, aplicado três vezes por semana, com duração de 40 minutos cada sessão. $\mathrm{O}$ aumento na área de secção transversa e a adição de sarcômeros em série no músculo sóleo de ratos, também foi observado no estudo de Secchi et al. ${ }^{30} \mathrm{O}$ protocolo utilizado foi aplicado por oito semanas de exercícios de alongamento estático, realizado 2 vezes por semana, com duração de 40 minutos.
Dessa forma, o alongamento estático estimula a síntese de proteínas e adição de sarcômeros em série ao longo da fibra muscular através da liberação de fatores de transcrição e de fatores de crescimento, como o IGF e o HGF, além de ativação de células satélites e aumento da síntese protéica e da expressão gênica como um todo. Todos estes fatores parecem estar envolvidos no processo de remodelação tecidual que resulta no aumento da flexibilidade ${ }^{28,30}$.

Apesar das divergências na explicação dos mecanismos responsáveis pelo aumento crônicos na amplitude de movimento, atividades que exigem altos níveis de flexibilidade por períodos longos, como a ginástica e a dança, podem gerar estímulos suficientes de modo que todos os mecanismos influenciem no resultado final da amplitude de movimento ${ }^{21}$.

Não foram encontrados, na literatura, artigos que comparassem a técnica Mulligan - Long Leg Traction com os alongamentos estáticos para o aprimoramento da flexibilidade. Entretanto, com base nas discussões apresentadas acima, observa-se que os mecanismos pelos quais ambos os grupos obtiveram aumento crônico na amplitude de movimento podem ser os mesmos.

\section{CONCLUSÃO}

Os resultados demonstraram que, após as seis semanas de treinamento, os dois métodos de alongamento estudados mostraram-se eficazes no aumento da amplitude de movimento de extensão do quadril de ginastas. Esses resultados possuem aplicação na prescrição de exercícios de alongamento, tanto para os períodos de treinamento quanto para os períodos competitivos da equipe em questão.

No entanto, apesar do contributo que se possa retirar deste estudo, é necessário ter em consideração que este estudo possui algumas limitações. Trata-se de uma série de casos com ginastas de uma única equipe, assim, não deve ser considerada representativa de toda a população de ginastas.

Sugere-se a realização de estudos semelhantes em um grupo maior de atletas de Ginástica Rítmica e de outros esportes. Seria também interessante levar em consideração o controle das variáveis que possam influenciar o estudo. E ainda, um delineamento metodológico de natureza longitudinal no sentido de avaliar a resposta da flexibilidade ao longo das diferentes fases e tipos de treinamento.

\section{REFERÊNCIAS BIBLIOGRÁFICAS}

1. Laffranchi B. Treinamento desportivo aplicado à ginástica rítmica. Londrina: UNOPAR; 2001. 
2. Dupont P. Medical Follow-Up and Lumbopelvic Flexibility in Rhythmic Gymnastics. J Traumatol Sport 2005;22(4):213-218.

3. Hutchinson MR. Low back pain in elite rhythmic gymnasts. Med Sci Sports Exerc. 1999; 31(11):1686-1688.

4. Roberts JM, Wilson K. Effect of stretching duration on active and passive range of motion in the lower extremity. Br J Sports Med. 1999;33(4):259-263.

5. Bandy WD, Irion JM. The effect of time on static stretch on the flexibility of the hamstring muscles. Phys Ther 1994;74(9):845-850.

6. Bandy, WD.; Irion JM., Briggler, M.The effect of time and frequency of static stretching on flexibility of the hamstring muscles.Phys Ther 1997;77(10):1090-1096.

7. Hall TM. Effects of the Mulligan traction straight leg raise technique on range of movement.J Man Manipulative Ther 2001;9(3):128-133.

8. Hall TM, Hardt S, Schäfer A, Wallin L. Mulligan Bent Leg Raise Technique: A preliminary randomised trial of immediate effects after a single intervention. Manual Therapy 2006;11(2):130-135.

9. Thomas JR, Nelson JK. Métodos de pesquisa em atividade física. 3.ed. Porto Alegre: Artmed, 2002.

10. Zonnenberg AJJ, Maanen CJ van, Elvers JWH, Oostendorp RAB. Intra/interrater reliability of measurements on body posture photographs. J

11. Braz RG, Goes FPDC, Carvalho GA. Confiabilidade e Validade de Medidas Angulares por Meio do Software para Avaliação Postural.FisioterMov 2008;21(3):117-126.

12. Mccreary EK, Provance, PG, Kendall FP, Rodgers M, Romani WA. Músculos: Provas e Funções. 5.ed. São Paulo: Manole, 2007.

13. Harvey D. Assessment of the Flexibility of Elite Athletes Using the Modified Thomas Test. Br J Sports Med 1998;32(1):68-70.

14. Taylor DC, Dalton JD, Seaber AV, Garrett WE. Viscoelastic properties of muscle-tendon units: the biomechanical effcts of stretching. Am J Sports Med1990;18(3):300-309.

15. Hartig DE, Henderson JM. Increasing Hamstring Flexibility Decreases Lower Extremity Overuse Injuries in Military Basic Trainees.Am J Sports Med 1999;27(2):173-176

16. Steinberg N, Hershkovitz I, Peleg S, Dar G, Masharawi Y, Heim M, et al. Range of Joint Movement in Female Dancers and Non Dancers Aged 8 to 16 Years: Anatomical and Clinical Implication.Am J Sports Med 2006;34(5):814-823.

17. Kirby RL, Simms FC, Symington VJ, Garner JB. Flexibility and Musculoskeletal Symptomatology in Female Gymnasts and Age-matched Controls.Am J Sports Med 1981;9(3):160-4.

18. L'Hermette M.; Polle G, Tourny-Chollet C, Dujardin F. Hip passive range of motion and frequency of radiographic hip osteoarthritis in former elite handball players. Br J Sports Med 2006;40(1):45-49.
19. Meszaros TF, Olson R, Kulig K, Creighton D, Czarnecki E.Effect of 10\%, 30\%, and 60\% Body Weight Traction on the Straight Leg Raise Test of-Symptomatic Patients with Low Back Pain. J Orthop Sports Phys Ther 2000;30(10):595-601.

20. Mosler AB, Blanch PD, Hiskins BC. The effect of manual therapy on hip joint range of motion, pain and eggbeater kick performance in water polo players. Phys Ther Sport 2006;7(3):128-136.

21. Magnusson SP, Simonsen EB, Aagaard P, Sorensen H, Kjaer M. A mechanism for altered flexibility in human skeletal muscle. J Physiol 1996;497(1): 291-298.

22. Davis DS, Ashby PE, McCale, KL, McQuain JA, Wine JM. The Effectiveness of 3 Stretching Techniques on Hamstring Flexibility Using Consistent Stretching Parameters. J Strength Cond Res 2005;19(1):27-32.

23. Winters MV. Passive versus active stretching of hip flexor muscles in subjects with limited hip extension: a randomized clinical trial. Phys Ther 2004;84(9):800-807.

24. Gajdosik RL. Effects of static stretching on the maximal length and resistance to passive stretch of short hamstring muscles. J Orthop Sports Phys Ther 1991;14(6):250-255.

25. DeDeyne PG. Application of passive stretch and its implications for muscle fibers.Phys Ther 2001;81(2):819-827.

26. Magnusson SP. Passive properties of human skeletal muscle during stretch maneuvers.Scand J Med Sci Sports1998;8(2):65-77.

27. Guissard N, Duchateau J. Neural aspects of muscle stretching. Exerc Sport Sci Rev 2006;34(4):154-158.

28. Menon TC, Casarolli LM, Cunha NB, Souza L, Andrade PHM, Albuquerque CE, et al. Influência do alongamento passivo em três repetições de 30 segundos a cada 48 horas em músculo sóleo imobilizado de ratos. Rev Bras Med Esporte 2007;13(6):407-410.

29. Coutinho EL, Gomes ARS, França CN, Oishi J, Salvini TF. Effect of passive stretching on the immobilized soleus muscle fiber morphology. Braz J Med Biol Res 2004;37(12):1853-1861.

30. Secchi KVI, Morais CPI, Cimatti PFI, Tokars EI, Gomes ARS. Efeito do alongamento e do exercício contra-resistido no músculo esquelético de rato. Rev Bras Fisioter 2008;12(3):228-234.

\section{Endereço para correspondência}

Manuela Karloh.

Associação Desportiva do Instituto Estadual

de Educação.

Avenida Mauro Ramos, 257,

CEP: 88020-30. Florianópolis, SC, Brasil..

E-mail: manukarloh@gmail.com 